\title{
Research of Condensate Resistance Measurement Using Single-Contact Substrate
}

\author{
Tomas Jukna ${ }^{1}$, Vytenis Sinkevicius ${ }^{1}$, Lina Urbanaviciute ${ }^{1,2}$, Jonas Valickas ${ }^{1}$ \\ ${ }^{I}$ Department of Technologies, Kaunas University of Technology, \\ Daukanto St. 12-103, LT- 35212 Panevezys, Lithuania \\ ${ }^{2}$ Department of Technology Science, Panevezys College, \\ Laisves Sq. 23-117, LT-35200 Panevezys, Lithuania \\ vytenis.sinkevicius@ktu.lt
}

\begin{abstract}
The research of non-invasive method for condensate resistance measurement uses a substrate with a single-contact area. The novelty of this measurement method lies in the use of electrical current of electrons emitted during the process of electronic emission from an evaporator. Therefore, there is no need of any additional current or voltage source. This study develops a mathematical model describing the performance and dependencies of this method of measurement. The adequacy of this mathematical model was investigated experimentally. For this purpose, the results of direct measurement of substrate condensate resistance were used.
\end{abstract}

Index Terms-Thin film devices, vacuum technology, electrical resistance measurement.

\section{INTRODUCTION}

In order to measure the thickness of conductive and semiconductive condensate layers in early formation stages, it is necessary to monitor and control condensate conductivity or resistance. The in-situ measurement (i.e. during layer formation stage) of conductive film surface resistance provides valuable information which helps to control the evaporation process and to determine the end point of realtime processes. Conductive film surface resistance can be measured by several methods.

The four-point probe method is one of the most widely used methods in the thin film resistivity measurement [1]. DC or AC voltage can be used in this method [2] and its value can be controlled by computer [3]. However, the contact dimension of the probe used, the distance between the contacts and the size of the test area affect the measurement results [4], [5]. During the measurement the probe is pressed against the plate part on which the conductive material is deposited, and this is the reason why this method is difficult to use in real time. The Van der Pauw measurement method is more suitable for real-time systems, as here four areas prepared for the contact are used. This method uses low $1.5 \mathrm{~V}-4.5 \mathrm{~V}$ voltage and the current of less than $1 \mu \mathrm{A}$ [6]. A transformer and a $1 \mathrm{kHz}$ frequency source can be used for galvanic isolation from the evaporator circuit [7]. This method is also suitable for the resistance measurement of ultra-thin films [8]. Space charge affects the results of resistance measurement during

Manuscript received 25 February, 2015; accepted 23 August, 2015. condensation and this influence must be eliminated [9]. On the other hand, the space charge can be used for resistance measurement [10] adapting low-energy electron beams [11], [12]. Therefore, it is preferred to measure the electrical resistance of vacuum-deposited condensates by a noninvasive method, which uses a flow of electrons emitted from the hot evaporator [13]. Experiments utilize direct stabilized voltage for evaporator heating. As the evaporator is powered from a direct current source, electromagnetic and electrostatic fields resulting from the heating current do not divert the electron flow away from the measurement probes and substrates. This helps to reduce the measured voltages and therefore, decreases the probability limits for the method under discussion. For our experimental research, we started to use additional electron source (electrically heated thin tungsten filament), which was installed near the evaporator [14].

For the comparison and analysis of experimental results, an additional direct measurement of the electrical resistance of vacuum-deposited condensates was applied. The setup consisted of a substrate with two contact areas and galvanically isolated source of stabilized current. This condensate resistance measurement method is based on the stabilized current, which flows through the substrate used to deposit the material being evaporated. However, resistance can be measured only after the saturation stage of the current stabilizer ends. Therefore, for this research, experiments involved reduced stabilized current allowing to maximize direct measurement limits for condensate resistance.

\section{THE EXPERIMENTAL ANALYSIS OF CONDENSATE RESISTANCE MEASUREMENT}

Experiments utilize three substrates P1, P2, and P3 located one beside the other (Fig. 1). The evaporator is heated by $5 \mathrm{~V}$ DC. An additional electron source is installed at the same height with the evaporator and is powered by $20 \mathrm{~V}$ DC. The experimental circuit diagram shows those two sources as an equivalent electromotive force (EMF) source $E$. Its voltage is measured by an additional circular metal probe $\mathrm{P}$ installed near the substrates. After setting the evaporation parameters, the shutter is opened and the vapour flow reaches the substrates. The resistance of substrate P1 is measured directly. By using AD210JN operational 
amplifier, substrate P1 is galvanically isolated from the system's common earth. The contact areas of P1 were connected to the stabilized current source $E_{S}$ (Fig. 1), which had the output voltage of $6.7 \mathrm{~V}$ during the experiments. The substrate had current $i_{s}$, and the voltage drop $U_{p 1}$ on the condensate of substrate P1 was measured during condensate growth.

Substrates P2 and P3 were intended to measure the resistance of the deposited condensate on them using electron emission current (non-invasive method). The substrate P3 has two contact areas; one of them is connected to the system's earth, and the other is connected to the data collection board (DCB) through INA128 operational amplifier installed within the vacuum chamber beside the substrate. The contact area of the substrate P2 is also connected to DCB through the operational amplifier. The substrate P2 has no second contact area. In the process of the experiment, substrate voltages $U_{p 2}$ and $U_{p 3}$ are measured respectively. Substrates $\mathrm{P} 2, \mathrm{P} 3$, and measurement probe $\mathrm{P}$ are connected to resistances $r_{m}=11 \mathrm{M}$.

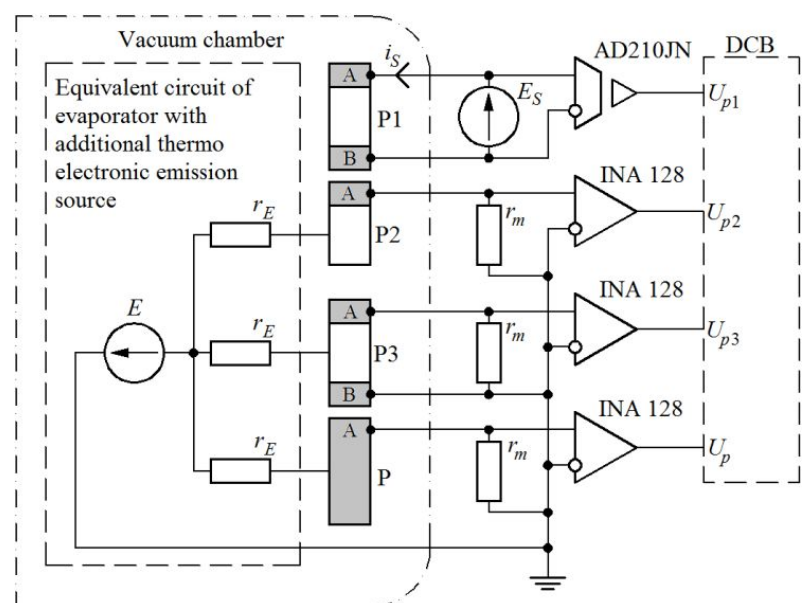

Fig. 1. Experimental circuit diagram for resistance measurement of growing condensate.

During five series of experiments, the stabilized current $i_{s}$ reduced from $0.6 \mu \mathrm{A}$ to $0.07 \mu \mathrm{A}$ in order to maximize the limits of direct measurement of condensate resistance. Figure 2 shows the experimental results at $i_{s}=0.07 \mu \mathrm{A}$.

The signals measured during experiments were filtered using the FFT simple window filter. To avoid a window edge effect, the signals were supplemented with mirror flipped reflection, and the middle up to $2^{\mathrm{N}}$ filled with a steady-state value. After filtering, all signals converted into positive values.

At $i_{s}=0,07 \mu \mathrm{A}$, experiments resulted in two times wider range of directly measured resistance compared to experimental results provided in [14], where $i_{s}$ was $2 \mu \mathrm{A}$. This also allowed to start measurements within the range of higher values of condensate resistance (in Fig. 2, section suitable for resistance measurement denoted as $H$ ).

Figure 2 shows voltages $U_{p 2}$ and $U_{p 3}$ of non-invasive measurement probes with one and two contacts. It is apparent that reduction of the stabilized current $i_{s}$ allowed to start direct measurement of the signal from the point, where an extreme signal appeared in the two-contact substrate. This helps to identify parameters of the mathematical model of non-invasive measurement method more precisely.
During experiment, non-invasive method of resistance measurements using two-contact substrate generated a bellshaped signal (Fig. 2). The extremum can be used for solving control tasks. However, each value of such signal voltage corresponds with two different values of condensate resistance.

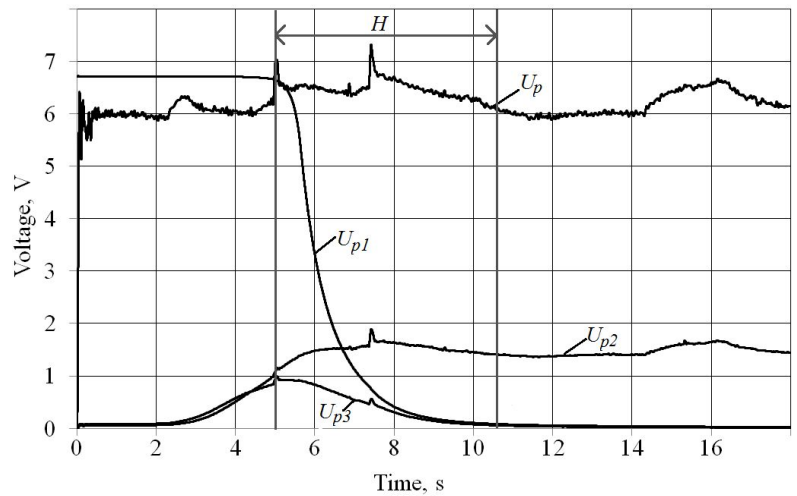

Fig. 2. Voltage changes in each one of the substrates and probe $\mathrm{P}$ during condensate growth.

It was noticed that a substrate with one contact area gives the signal identical to that of a two-contact substrate at the initial stage of the condensation process. Later, this signal continues to increase. After the extremum appears in the two-contact substrate, voltage increase in the single-contact substrate starts to slacken at the same time. Therefore, this signal can be unambiguously related to the condensate resistance.

\section{MATHEMAtical Model of CONDENSATE Resistance MEASUREMENT OF THE SubSTRATE WITH Single-CONTACT AREA}

Origin of the signal $U_{p 2}$ (Fig. 2) in a single-contact substrate can be explained by a mathematical model, developed in this section of the paper.

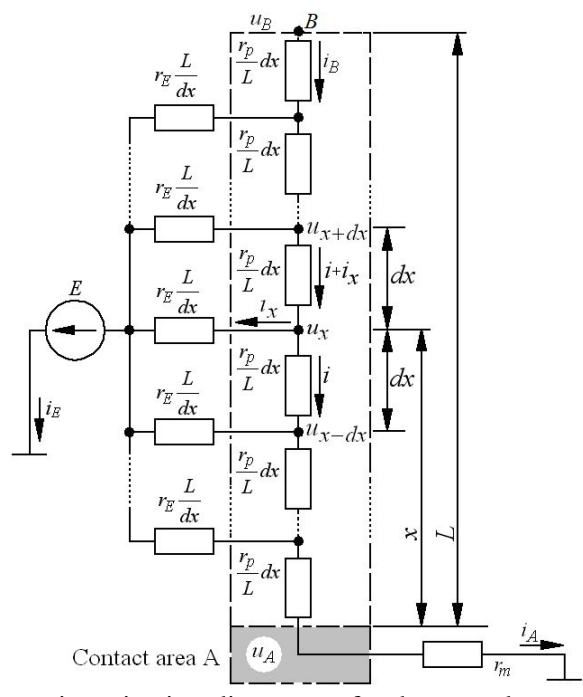

Fig. 3. Schematic circuit diagram of the condensate resistance measurement model, where contact area $\mathrm{A}$ is grounded through the measurement resistance $r_{m}$, and there is no area $\mathrm{B}$.

Structure of the condensate resistance measurement probe and the principle of condensate growth is the same as it was in the previous model of growing condensate resistance measurement [15], just contact area $\mathrm{A}$ of the probe is 
grounded through the measurement resistance $r_{m}$, and there is no area B (Fig. 3). Surface area of this contact area in a model is assumed to be zero, i.e. its impact is not evaluated. As there are no other current sources in this circuit, it means that $i_{A}=i_{E}$.

Condensate resistance is denoted as $r_{p}$ in the mathematical model. For model development, condensate strip is divided into sub-strips of the length $d x$ (Fig. 3). The equivalent resistance of the electric charge flow $i_{E}$ denoted as $r_{E}$. During the model development, electromotive forces of both the evaporator and the additional electron source were taken as equivalent EMF, which denoted as $E$.

Potential $u_{x}$ of the sub-strip with width $d x$ relates to the distance $x$ from the area A as follows

$$
\frac{d^{2} u_{x}}{d x^{2}}-\frac{r_{p}}{r_{E} L^{2}} u_{x}=-\frac{r_{p}}{r_{E} L^{2}} E .
$$

Characteristic roots of the differential equation (1) are as follows

$$
\lambda_{1,2}= \pm \frac{1}{L} \sqrt{\frac{r_{p}}{r_{E}}}= \pm \frac{\beta}{L}
$$

Common solution of the differential equation (1) is as follows

$$
u_{x}=C_{1} e^{x \beta / L}+C_{2} e^{-x \beta / L}+E .
$$

Expression of the current $i_{x}$ in the substrate has also been obtained

$$
i_{x}=\beta / r_{p} \times\left(C_{1} \times e^{x \beta / L}-C_{2} \times e^{-x \beta / L}\right)
$$

Coefficients $C_{1}$ and $C_{2}$ depend on initial conditions in the contact area A. In this case, the distance $x=0$ and $u_{x}=u_{A}$ (Fig. 3, Fig. 4) because potential of this area equals voltage drop on the resistance $r_{m}$, which has a current $i_{A}$. Then, initial conditions obtained from (3) are as follows

$$
-i_{A} \times r_{m}=C_{1}+C_{2}+E
$$

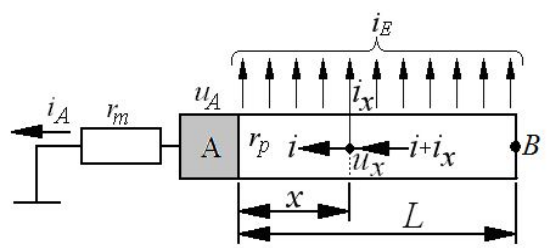

Fig. 4. Generalized schematic circuit diagram of the condensate resistance measurement model.

After finding current $i_{A}$ from (4) at $x=0$ and from (5), these values are equalized, and the following is obtained

$$
-E=C_{1} \times\left(1-\beta \times r_{m} / r_{p}\right)+C_{2} \times\left(1+\beta \times r_{m} / r_{p}\right) .
$$

Initial conditions at the other end of the substrate with no contact area in this case are found from the expression of the current $i_{x}$ (4). There is no current at the point B (Fig. 3), located at the distance $x=L$ from the area A. Therefore, it is possible to write (4) as follows

$$
i_{B}=\beta / r_{p} \times\left(C_{1} \times e^{\beta L / L}-C_{2} \times e^{-\beta L / L}\right)=0 .
$$

Current $i_{B}(7)$ becomes zero only when

$$
C_{1} \times e^{\beta}-C_{2} \times e^{-\beta}=0 \text {. }
$$

To find coefficients $C_{1}$ and $C_{2}$ from (6) and (8), system of equations is made:

$$
\left\{\begin{array}{rl}
0 & =C_{1} \times e^{\beta}-C_{2} \times e^{-\beta}, \\
-E & =C_{1} \times\left(1-\beta \times r_{m} / r_{p}\right)+C_{2} \times\left(1+\beta \times r_{m} / r_{p}\right) .
\end{array} .\right.
$$

After finding coefficients $C_{1}$ and $C_{2}$ from (9) and inserting them into (3), expression of the condensate potential $u_{x}$ is obtained

$$
\begin{aligned}
u_{x}= & \frac{-E}{\left(e^{\beta}+e^{-\beta}\right)+\beta \times r_{m} / r_{p} \times\left(e^{\beta}-e^{-\beta}\right)} \times \\
& \times\left(e^{-\beta / L \times(L-x)}+e^{\beta / L \times(L-x)}\right)+E .
\end{aligned}
$$

Equation (10) is the expression of the potential $u_{A}$ of the contact area $\mathrm{A}$ at the distance value $x=0$ and $r_{p}=\beta^{2} r_{E}$

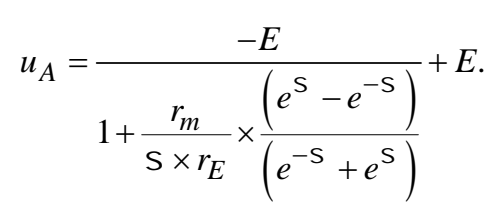

Later, a test was carried out to determine how potential changes of $u_{A}$ relate to the condensate resistance $r_{p}$. The values of condensate voltage $u_{A}$ are checked both at the initial and final stages of the condensate growth, i.e. when the condensate resistance value $r_{p}$ is infinity and zero:

$$
\left\{\begin{array}{l}
\lim _{r_{p} \rightarrow \infty}\left(u_{A}\right)=0, \\
\lim _{r_{p} \rightarrow 0}\left(u_{A}\right)=E r_{m} /\left(r_{E}+r_{m}\right) .
\end{array}\right.
$$

Looking at (12), it becomes obvious that equivalent internal resistance of both thermo-electronic emission and vacuum part can be found from the voltage expression $u_{A}$, when condensate resistance approaches to zero

$$
r_{E}=r_{m} \times\left(E / u_{A}-1\right)
$$

During condensation, resistance $r_{E}$ is not constant and significantly depends on the distribution of melted material on the evaporator surface. Equation (13) is to be applied just at the final stage of condensation, when condensate resistance decreases and its value becomes of the order of ohms. To monitor changes of this resistance during the whole experiment, a metal probe $\mathrm{P}$ (Fig. 1) is installed, and the voltage $U_{p}$ is measured. Then, resistance $r_{E}$ during the condensation process can be calculated as follows 


$$
r_{E}(t)=r_{m} \times\left(E / u_{P}(t)-1\right) .
$$

During the experiment, condensate resistance $r_{p}(t)$ was measured using direct measurement method to check the mathematical model of the condensate resistance. However, due to limited power voltage of the current source, this method may only be applied after the saturation stage of the stabilized current source ends. To check adequacy of the model, it was needed to find coefficients $a_{0}, a_{1}$, and $a_{2}$ of the function, which interpolates resistance changes within the range from $t_{1}$ to $t_{2}$ (Fig. 5)

$$
r_{p}^{*}(t)=\exp \left(a_{0}+a_{1} \ln (t)+a_{2} \ln ^{2}(t)\right)
$$

In order to compare the changes of the single-contact substrate voltage, $u_{A}$ was calculated using (11), (14), and (15). Then

$$
u_{A}(t)=\frac{-E}{1+\frac{r_{m}}{\beta \times r_{E}(t)} \times \frac{\left(e^{\beta}-e^{-\beta}\right)}{\left(e^{-\beta}+e^{\beta}\right)}}+E,
$$

where

$$
\beta=\sqrt{r_{p}^{*} / r_{E}(t)}
$$

Figure 6 shows voltage $U_{p 2}$ measured during the experiment, and voltage $u_{A}$ calculated using (16).

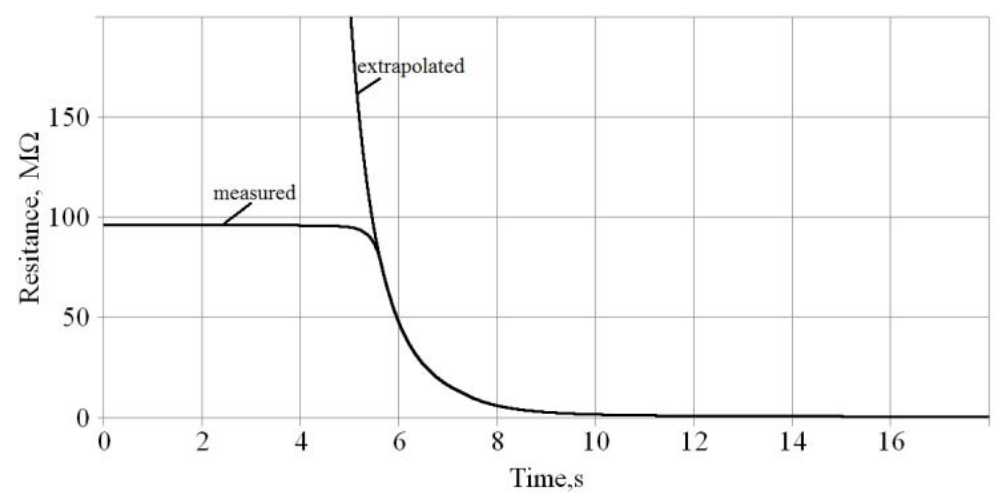

Fig. 5. Condensate resistance both extrapolated and measured using an electric current method.

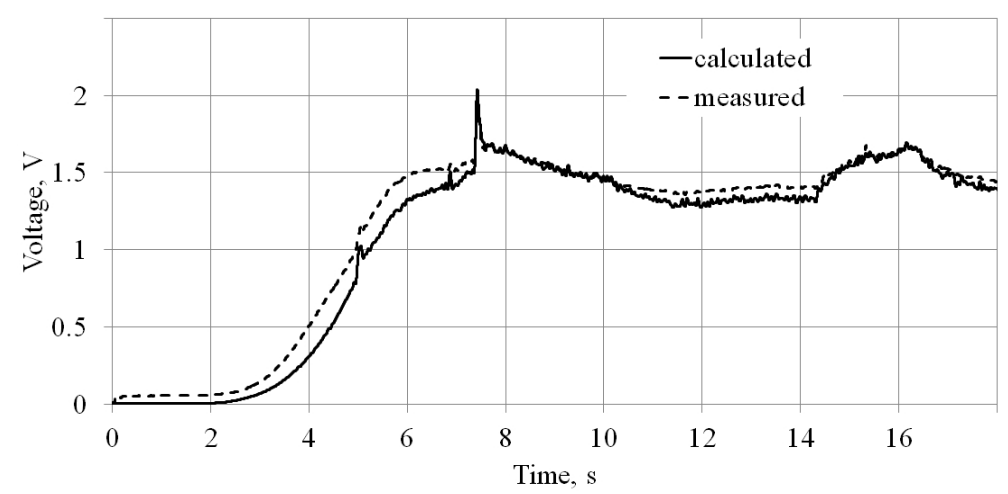

Fig. 6. Single-contact substrate voltage changes both measured and calculated.

Substrate contact potential measured at the initial stage of the condensation process was not zero (Fig. 6). This can be explained by the electrons from thermo-electronic emission constantly hitting the finite surface area of the contact area. This results in a steady component of the voltage. A mathematical model does not evaluate the surface area of this contact area. Condensate resistance in a section starting from the point $t_{1}$ is measured, and therefore, its value is known. However, the probe, whose signal was used to calculate $r_{E}(t)$ was placed near the substrate P2. This might have caused calculation inaccuracies for $r_{E}(t)$.

\section{CONCLUSIONS}

1. A non-invasion measurement method using a probe with one or two contacts, makes it possible to measure condensation resistance earlier than this can be done using the traditional method.

2. In case of a non-invasion measurement method, when a probe with a single contact is used, the signal monotonically increases until the steady value and is unambiguously associated with the condensate resistance of the tray.

3. The mathematical model of this method of measurement demonstrated that during the measurement time it is necessary to assess the changing-in-time equivalent internal resistance of electronic thermal emission and its vacuum section.

4. Within the mathematical model it is necessary to evaluate the area of contact plates and thus eliminate the constant component of the signal.

\section{REFERENCES}

[1] Sh. Yoshimoto, Y. Murata, et al, "Four-point probe resistance measurements using PtIr-coated carbon nanotube tips", Nano Lett., vol. 7, no. 4, pp. 956-959, 2007. [Online]. Available: http://dx.doi.org/10.1021/n10630182

[2] K. Brian, Z. Wang, et al, "Generalized four-point characterization method using capacitive and ohmic contacts", Review of Scientific Instruments, vol. 83, no. 2, pp. 24703-11, 2012. [Online]. Available: http://dx.doi.org/10.1063/1.3677331 
[3] P. Haibin, D. Jianning, W. Xiaofei, L. Boquan, "Design, implementation, and assessment of a highprecision and automation measurement system for thin film resistivity", in Int. Conf. (MACE 2010), 2010, pp. 2235-2238.

[4] Yanqun Wang, P. H. Schimpf, et al, "Geometric effects on resistivity measurements with four-electrode probes in isotropic and anisotropic tissues", IEEE Trans. Biomedical Engineering, vol. 45, no. 7, pp. 877-884, 1998. [Online]. Available: http://dx.doi.org/10.1109/10.686795

[5] K. Ilse, T. Tanzer, C. Hagendorf, M. Turek, "Geometrical correction factors for finite-size probe tips in microscopic four-point-probe resistivity measurements", Journal of Applied Physics, vol. 116, no. 22, pp. 224509-7, 2014. [Online]. Available: http://dx.doi.org/ $10.1063 / 1.4903964$

[6] M. Morvic, "On the measurement of high resistance semiconductors by the van der Pauw method", in Advanced Semiconductor Devices and Microsystems 2000, pp. 327-330, 2000. [Online]. Available: http://dx.doi.org/10.1109/asdam.2000.889512

[7] E. V. Barnat, T.-M. Lu, "Real time copper resistivity measurements during sputter deposition”, in Interconnect Technology Conf., 2001 , pp. 24-26. [Online]. Available: http://dx.doi.org/10.1109/iitc.2001. 930005

[8] E. V. Barnat, D. Nagakura, et al, "Real time resistivity measurements during sputter deposition of ultrathin copper films", Journal of Applied Physics, vol. 91, no. 3, pp. 1667-1672, 2002. [Online] Available: http://dx.doi.org/10.1063/1.1430530
[9] B. R. Medina, A. P. Estrada, F. H. Marquez, "Improvements in high resistance measurements at CENAM", in Precision Electromagnetic Measurements, pp. 358-359, 2012. [Online]. Available: http://dx.doi.org/10.1109/cpem.2012.6250950

[10] J. R. Dennison, J. Brunson, et al, "Methods for high resistivity measurements related to spacecraft charging", IEEE Trans. Plasma Science, vol. 34, no. 5, 2006. [Online]. Available: http://dx.doi.org/ 10.1109/TPS.2006.883400

[11] A. N. Chester, B. B. Kosicki, "An electron beam method for measuring high sheet resistances of thin films", Review of Scientific Instruments, vol. 41, no. 12, pp. 1817-1824, 1970. [Online]. Available: http://dx.doi.org/10.1063/1.1684418

[12] A. Czerwinski, M. Pluska, J. Ratajczak, A. Szerling, "Resistance and sheet resistance measurements using electron beam induced current", Applied Physics Letters, vol. 89, no. 24, pp. 241919-3, 2006. [Online]. Available: http://dx.doi.org/10.1063/1.2405886

[13] V. Sinkevicius, "Research of the method opportunities of growing films conductance measurement", Elektronika ir Elektrotechnika, no. 4, pp. 71-74, 2009.

[14] T. Jukna, V. Sinkevicius, L. Urbanaviciute, "Influence of emitted electrons on the method for direct measurement of condensate resistance", Elektronika ir Elektrotechnika, no. 2, pp. 28-31, 2014. [Online]. Available: http://dx.doi.org/10.5755/j01.eee.20.2.6379

[15] V. Sinkevicius, "The mathematical model of conductance measurement in growing thin films", Elektronika ir Elektrotechnika, no. 5 , pp. 17-20, 2008 